\title{
Robotic Systems in Radiotherapy and Radiosurgery
}

\author{
Stefan Gerlach ${ }^{1}\left[\mathbb{D}^{-}\right.$Alexander Schlaefer ${ }^{1}$
}

Accepted: 6 December 2021 / Published online: 8 January 2022

(c) The Author(s) 2022

\begin{abstract}
Purpose of Review This review provides an overview of robotic systems in radiotherapy and radiosurgery, with a focus on medical devices and recently proposed research systems. We summarize the key motivation for using robotic systems and illustrate the potential advantages.

Recent Findings. Robotic systems have been proposed for a variety of tasks in radiotherapy, including the positioning of beam source, patients, and imaging devices. A number of systems are cleared for use in patients, and some are widely used, particularly for beam and patient positioning.

Summary The need for precise and safe delivery of focused high doses to the target region motivates the use of robots in radiotherapy. Flexibility in the arrangement of beams and the ability to compensate for target motion are key advantages of robotic systems. While robotic patient couches are widely used and robotic beam positioning is well established, brachytherapy robots are mostly considered in a research context.
\end{abstract}

Keywords Robotic radiation therapy $\cdot$ Robotic radiation surgery $\cdot$ Robotic brachytherapy $\cdot$ Robotic patient positioning $\cdot$ Robotic ultrasound tracking

\section{Introduction}

With an incidence of over 19 million in 2020 [1], cancer is one of the leading causes of death. Radiotherapy (RT) has long been one of the most widely used and effective treatment options, which is required in over $60-70 \%$ of these cases [2]. However, since the ionizing radiation that is at the core of the treatment can also impose severe harm to patients and staff, safe and automated dose delivery is one of the key challenges of RT. Another interesting aspect is the localization of the target, which is typically deep inside the patient and not readily visible. It may also be subject to patient motion which has to be considered during treatment. Given the challenging environment, the need for automation, and the dynamics of patient motion, a number of systems

Stefan Gerlach

stefan.gerlach@tuhh.de

Alexander Schlaefer

schlaefer@tuhh.de

1 Institute of Medical Technology and Intelligent Systems, Hamburg University of Technology, Am Schwarzenberg-Campus 3, 21073 Hamburg, Germany based on robots and robotics methods have been proposed. The steady increase in the numbers of publications shown in Fig. 1 illustrates the growing interest in robotic systems in RT.

Conventionally, a gantry mounted beam source, rotating about the patient, delivers the desired dose in external beam RT. While some systems based on radioactive sources (Cobalt) exist (e.g., GammaKnife), most external beam treatment devices are based on linear accelerators (LINACs). Electron beam or Proton and heavy ion-based treatment devices present an interesting alternative with different dose absorption patterns (e.g., Bragg-peak), which allows controlling the dose along the beam inside the patient. In contrast, the more widely used photon-beam systems rely on beams from different directions to create a high-dose region in the target. Precise treatments with steep dose gradients around the target such that the high doses conform to the target shape are often referred to as radiosurgery (RS).

An interesting alternative to external beam RT is brachytherapy (BT). Typically, radioactive sources placed directly in the target region are used for irradiation. Lowdose sources are delivered as small seeds and remain in the patient. Alternatively, high-dose sources are moved through hollow needles or tubes after these have been placed in the 


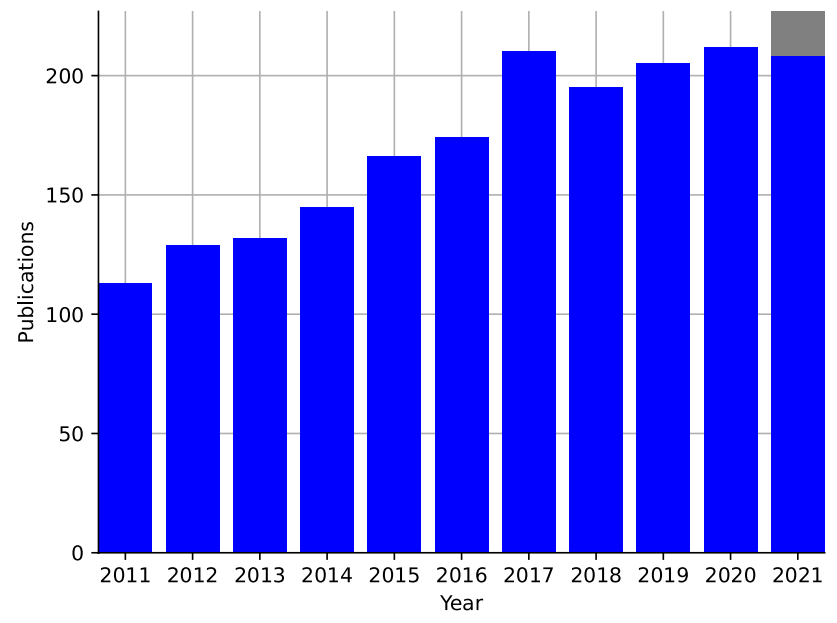

Fig. 1 Publications of papers related to robotics in RT or RS indexed on PubMed. Search term: "robotic and (radiosurgery or radiotherapy)." Publications in blue; linear extrapolation in grey

target. Conventionally, needles are placed manually by the physician using a template guiding needle insertion.

A general challenge with all forms of RT is the conflicting objectives when delivering the dose. Namely, there is a need to deliver a high, effective dose to the target structures to control the tumor, while at the same time limiting the dose to other, healthy structures as much as possible. Inverse planning is typically used to derive the treatment parameters corresponding to the best clinical trade-off. Initially, the three-dimensional shape of the target and relevant healthy structures called organs-at-risk (OAR) is delineated. The uncertainty with respect to the dose delivery needs to be estimated and accounted for by adding margins to form the planning target volume (PTV) and the planning risk volumes (PRV). However, adding the margins will generally further aggravate the conflicts with respect to the objectives and margins around the target will also subject healthy tissue to high doses. Hence, to keep the margins related to uncertainty in the setup and the pose of the target low, image guidance with X-ray imaging, magnetic resonance imaging (MRI), or ultrasound (US) is widely used.

\section{Clinical Motivation for Robotics}

As indicated, there are a number of practical concerns which make RT an interesting application area for robotics methods. First, the ionizing radiation requires particular safety measures for patients and staff. Typically, the staff has to be shielded from the radiation and cannot be in the direct proximity of the patients during treatment. Changes with respect to the patient's pose or the configuration of the treatment device need to be controlled remotely. Hence, even more conventional treatment couches and gantries can be considered kinematic structures similar to robots.

Second, the treatment needs to be focused on the target as much as possible in order to avoid potentially serious side effects. Precise placement of beams or seeds is important to shape a high-dose region that effectively covers the target. Misalignments can cause deviations from the desired dose often referred to as cold and hot spots which could ultimately compromise the treatment or lead to side effects. The flexibility in beam or seed placement offered by robotic systems can improve dose distributions to match the patient's anatomy more closely as illustrated in Fig. 2. The figure motivates that using beams from several different directions can help shaping the dose distribution. Note, that the advantage of robotic systems is that they can place beams with a large solid angle, i.e., in three-dimensional space. Furthermore, the figure illustrates that precise needle insertions at different angles may offer advantages like fewer penetrations of the perineum in prostate BT.

Third, the dose delivery is a function of time and, particularly in external beam RT, relative motion between the beams and the target can occur while the beams are active. Common causes include spontaneous patient motion and physiological motion, particularly due to breathing. The latter is interesting as it cannot be avoided but can be substantial, e.g., in areas close to the diaphragm. As shown in Fig. 3, robots can be used to either move the beams synchronously with the target or to move the patient accordingly to compensate for the motion of the target.

However, to first localize the target and to establish its motion, it is necessary to employ some form of image guidance. Again, robots can assist in accurate positioning of imaging systems to not interfere with the RT treatment as shown in Fig. 4, where a robotic C-arm for intra-treatment $\mathrm{X}$-ray imaging and a robotic ultrasound system for continuous tracking of motion in the abdomen are illustrated.

In summary, robots can be used in areas subject to radiation, they can be used to precisely align beams or seeds, they can compensate target organ motion by synchronous movements, and they can be employed to realize precise, repeatable imaging. Subsequently, we will review recent work in all of these directions.

\section{Overview of Robotic Systems in Radiotherapy and Radiosurgery}

In each of the following chapters, we give an overview of interesting and relevant recent research and commercial systems as well as a brief summary of the historical development. We mainly limit this review to publications on RT and RS with linear accelerators as devices for treatment delivery for external RT and only briefly touch on works on proton 
Fig. 2 Robot-based systems can offer additional flexibility for RT delivery. Top: Illustration of the effect of adding more beam directions on the conformality of the prescribed dose (red). More beam directions typically allow fitting the prescribed dose closer to the target shape. Note that the actual advantage of robotic systems is the threedimensional arrangement of beams. Bottom: Illustration of a potential benefit of using oblique needle trajectories through a single entry point as opposed to the more conventional template-guided parallel needles
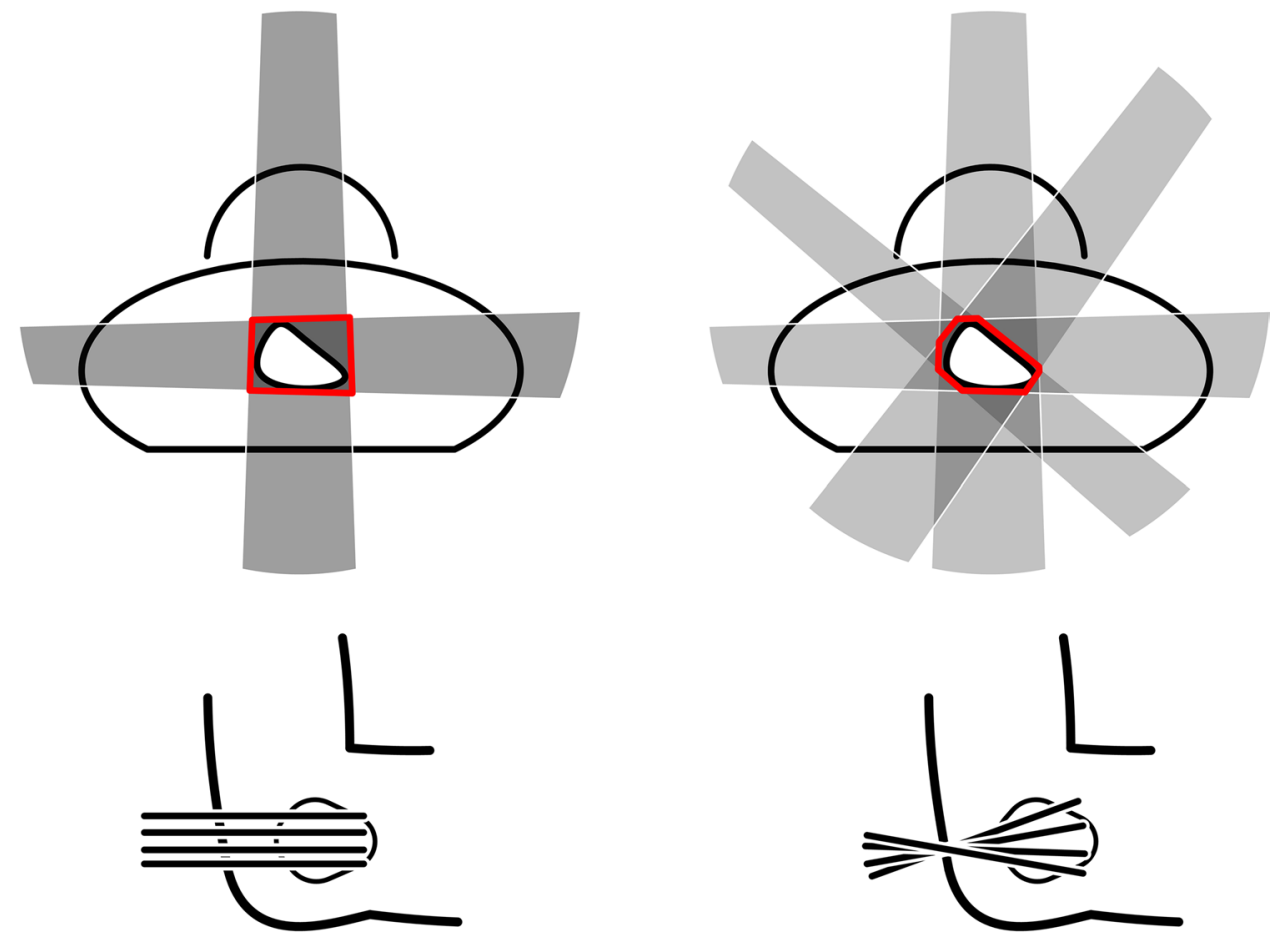
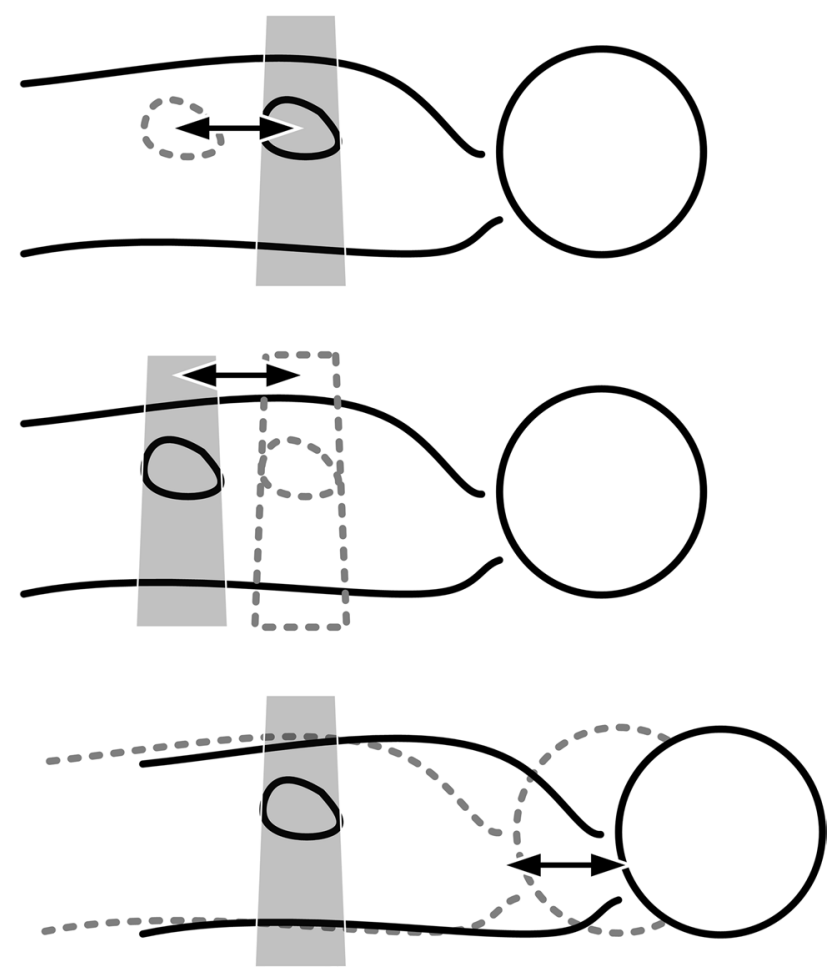

Fig. 3 Illustration of robotic motion compensation. Top: A typical situation during treatments in thorax and abdomen is the motion of the target due to breathing, i.e., the target is repeatedly leaving its initial position shown in black towards the dashed position and back, as shown by the arrow. Middle: Robotic systems can be used to compensate the motion of the target by moving the beams synchronously. Bottom: The same effect can in principle be reached by moving the patient such that the target remains within the beam or heavy ion therapy. Moreover, we will primarily discuss systems and approaches that have been covered in research papers over the past 5 years as well as highly relevant earlier works and commercial systems. Table 1 summarizes the systems.

\section{Robotic Beam Positioning}

The development of the first beam positioning using a 6 DoF robot dates back to 1992, when a predecessor of the CyberKnife (Accuray, Sunnyvale, USA) was presented by John Adler [40]. While the device was initially designed with a focus on neurosurgical treatments and precise, highly focused dose delivery, it has evolved into a versatile system for RS and RT [3••]. The recent version includes a 1000 MU/min $6 \mathrm{MeV}$ LINAC mounted to a $6 \mathrm{DoF}$ serial robot, a stereo X-ray camera system, and either a standard or robotic treatment couch. Beam apertures are shaped by either fix cylinder collimators, an IRIS collimator, or a multi-leaf collimator (MLC). Optional equipment includes an optical tracking system for respiratory motion compensation.

With a wide range of clinical applications, different objectives need to be considered [41•]. While superior conformality of the dose distribution is particularly important for tumors in the brain or spine, the treatment time or the ability to compensate motion may be more relevant for other targets. Note that generally a large number of beams which cover part of the PTV are used and individually weighted. From a planning perspective, more beams with smaller 
Fig. 4 Illustration of robotic approaches for image guidance in RT. Left: Robotic C-arms have been used in numerous clinical applications, including BT. Right: Robotic ultrasound has been proposed for continuous image guidance during external beam RT. The robot allows adjusting the ultrasound probe at the patient while the beam is active
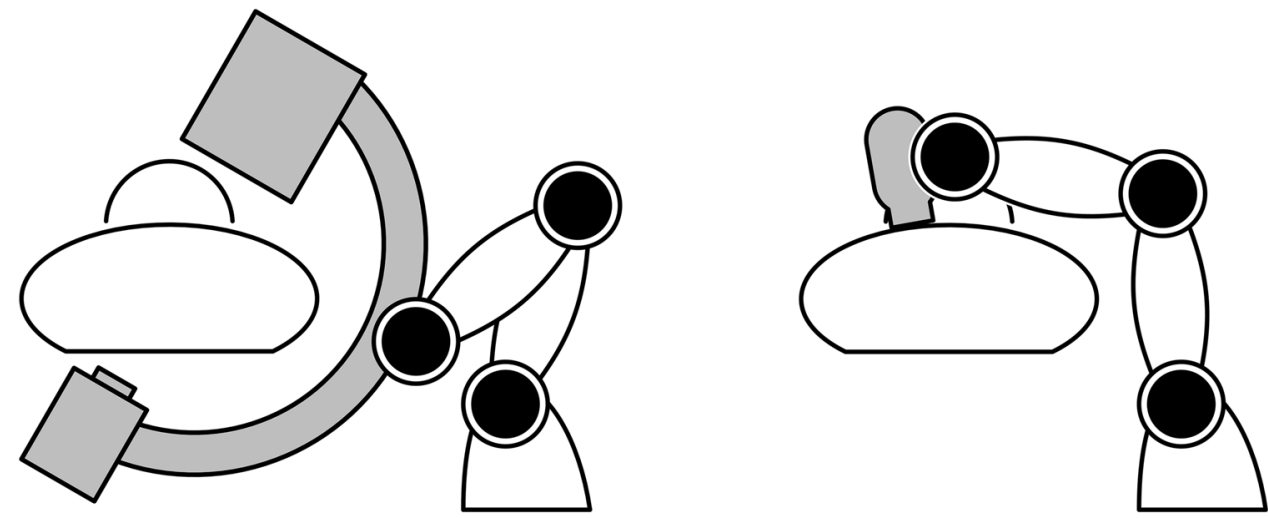

apertures add flexibility in shaping the dose distribution while larger apertures deliver more dose at once and tend to result in more efficient plans with fewer beams. Recent work addresses the time required for robot motion by studying approaches for continuous dose delivery while moving the LINAC [42, 43]. Clearly, this involves a trade-off between treatment time, number of different beam directions, and complexity of the beam apertures.

For targets that are subject to substantial motion, the ability of the robot to move the beam source rather rapidly has enabled a completely new treatment paradigm, i.e., to move the beams to account for any target motion that is detected during treatment [44]. This is particularly interesting for targets moving due to respiration, e.g., in the lower thorax and upper abdomen. These targets typically exhibit cyclic motion which can be predicted to control the robot movements accordingly [45]. The CyberKnife is routinely used for motion compensated treatments.

A device with similar freedom in using non-coplanar beams and active beam motion for motion compensation was the Vero System (Mitsubishi Heavy Industries, Tokyo, Japan, and BrainLAB, Munich, Germany). The device was based on a gimbaled LINAC mounted on a rotatable gantry which also integrated X-ray imaging. However, marketing for the system has stopped due to a commercial decision [46•].

More recently, another device based on a gyroscopic LINAC has been developed. The ZAP-X (ZAP Surgical Systems, San Carlos, USA) [7, 8] is designed for cranial RS and addresses the high initial cost of a radiation proof bunker which is necessary for conventional RT. The system is based on a self-shielded approach and can be operated without a bunker. During treatment, the LINAC can be moved with 2 DoF on almost the complete sphere around the iso-center. The integrated couch aligns the target with the iso-center before and during treatment with $3 \mathrm{DoF}$.

Another system currently in development is the Flashknife (PMB Alcen, Peynier, France) using the Oriatron eRT6 LINAC [4]. Flash RT uses brief high intensity "flashes" of radiation to treat cancerous tissue. Flash therapy relies on an increased reaction of cancerous tissue to high-dose rates while reaction of normal tissue is minimal. The Flashknife system consists of a LINAC mounted to a 6 DoF serial robot which is placed on a moving platform. The LINAC is hand-guided to the target by the physician. The system has successfully completed the first human trial [5].

Intraoperative electron radiotherapy (IOERT) represents an additional option for external RT. In IOERT, electron beams deliver dose to the surface level or surgically exposed tumor. The commercially available Liac and Novac devices (Sordina IORT Technologies, Vicenza, Italy) allow automatic positioning of the electron accelerator based on optical markers and treatment planning [10]. Due to low scatter radiation, the system does not require any additional shielding and can be integrated in a conventional operating room.

While research in novel robotic systems for RT or RS delivery is rare from non-commercial parties due to the costs associated with prototypes, Noh et al. presented a prototypical ceiling mounted 5 DoF system for delivery of cylinder shaped beams [11]. The robotic system was designed and simulated such that the workspace excludes poses near the patient couch and a prototype was manufactured.

\section{Robotic Patient Positioning}

Patient couches offering several DoF have been used to support the setup in external beam RT for quite some time. Several 6 DoF robotic couches are now used in external RT and RS to reproduce positioning of the patient either automatically or manually from the planning imaging for treatment such that the target structures align with their planned pose relative to the treatment system. For gantry-based RT and particularly for proton or heavy ion therapy, positioning the patient is important, since fewer DoF are available for aligning the beams.

Moreover, it has been established that $6 \mathrm{DoF}$ patient couches offer advantages with respect to treatment accuracy 
Table 1 Overview of recently developed and commercial robotic systems in RT and RS

\begin{tabular}{|c|c|c|c|c|c|c|}
\hline Group/name & Type & $\begin{array}{l}\text { Commercial avail- } \\
\text { ability }\end{array}$ & DoF & Kinematic & Clinical readiness & Acc $[\mathrm{mm}]$ \\
\hline Cyberknife $[3 \bullet \bullet]$ & Beam positioning & Accuray & 6 & Serial & FDA,CE & $<0.95[3 \bullet \bullet]$ \\
\hline FLASHKNiFE $[4,5]$ & Beam positioning & PMB Alcen & $6+3$ & Serial+ platform & 1 patient & n.r \\
\hline Vero & Beam positioning & $\begin{array}{l}\text { Mitsubishi Heavy } \\
\text { Industries + Brain- } \\
\text { LAB }\end{array}$ & 4 & Gimbaled gantry & FDA,CE & $<1[6]$ \\
\hline $\mathrm{ZAP}[7,8]$ & Beam positioning & Zap Surgical Systems & $2+3$ & $\begin{array}{l}\text { Gyroscopic + serial } \\
\text { couch }\end{array}$ & FDA,CE & $<1[9]$ \\
\hline $\begin{array}{l}\text { Liac/Novac devices } \\
\text { [10] }\end{array}$ & Beam positioning & SIT & $5+3$ & Serial + platform & FDA,CE & n.r \\
\hline Noh et al. [11] & Beam positioning & No & 5 & Serial & Phantom & n.r \\
\hline RoboCouch & Patient couch & Accuray & 6 & Serial & FDA,CE & 0.1 (rep. acc.) [12] \\
\hline $\begin{array}{l}\text { Standard Treatment } \\
\text { Couch }\end{array}$ & Patient couch & Accuray & 5 & Serial & FDA,CE & 0.3 (rep. acc.) [12] \\
\hline PreciseTable & Patient couch & Elekta & 3 & Serial & FDA,CE & $0.43[13]$ \\
\hline HexaPod & Patient couch & Elekta & 6 & Parallel & FDA,CE & $<0.5[14]$ \\
\hline PerfectPitch & Patient couch & Varian & 6 & Serial & FDA,CE & $0.7[15]$ \\
\hline Orion & Patient couch & Leoni & 6 & Serial & FDA,CE & $0.5[16]$ \\
\hline Examove & Patient couch & $\mathrm{BEC}$ & $6(+1)$ & $\begin{array}{l}\text { Serial (+ optional } \\
\text { linear rail) }\end{array}$ & FDA,CE & $0.5[17]$ \\
\hline Protura & Patient couch & Civco & 6 & Serial & FDA,CE & $<1[18]$ \\
\hline Belcher et al. [19] & $\begin{array}{l}\text { Head motion com- } \\
\text { pensation }\end{array}$ & No & 6 & Parallel & Pre-clinical & $99 \%<0.5 \mathrm{~mm}$ \\
\hline Ogunmolu et al. [20] & $\begin{array}{l}\text { Head motion com- } \\
\text { pensation }\end{array}$ & No & 6 & Parallel & Simulation & n.r \\
\hline Liu et al. [21] & $\begin{array}{l}\text { Motion compensa- } \\
\text { tion }\end{array}$ & No & 6 & Parallel & Pre-clinical & $100 \%<0.5 \mathrm{~mm}$ \\
\hline $\begin{array}{l}\text { Ostyn et al. }[22 \bullet \bullet \text {, } \\
23]\end{array}$ & Head positioning & No & 6 & Parallel & Phantom & 0.7 \\
\hline Hua et al. [24] & CT system & No & 6 & Serial & Clinical routine & $<0.56$ \\
\hline Schlosser et al. [25] & US motion tracking & No & 9 & Serial & Pre-clinical & n.r \\
\hline Ipsen et al. [26] & US motion tracking & No & 7 & Serial & Pre-clinical & n.r \\
\hline Sen et al. [27] & US patient setup & No & 6 & Serial & Phantom & 1.8 \\
\hline Seitz et al. [28] & US motion tracking & No & 7 & Serial & Pre-clinical & n.r \\
\hline First [29] & $\mathrm{BT}$ & Nucletron, Elekta & $2+1$ & Serial + needle driver & FDA,CE & $<1 \mathrm{~mm}$ (patient) \\
\hline Adams et al. $\left[30^{\bullet}\right]$ & $\mathrm{BT}$ & No & $5+1$ & $\begin{array}{l}\text { Serial + needle rota- } \\
\text { tion }\end{array}$ & Pre-clinical & 0.18 (air) \\
\hline Dou et al. [31] & BT & No & 4 & Serial & Patient trials [32] & 1.5 (phantom) \\
\hline $\begin{array}{l}\text { Fanhao et al./Reme- } \\
\text { bot [33] }\end{array}$ & BT & Remebot & $6+3$ & Serial + platform & 3 patients & 1.9 (patient) \\
\hline Jiang et al. [34] & BT & No & $3+2+4$ & $\begin{array}{l}\text { Serial plat- } \\
\text { form + tem- } \\
\text { plate + US probe }\end{array}$ & Pre-clinical & 0.7 (air) \\
\hline Lin et al. [35] & BT & No & $5+1$ & Serial + needle driver & Pre-clinical & 0.69 (air) \\
\hline Ma et al. [36] & BT & No & 6 & Serial & Animal & 1.04 (phantom) \\
\hline Belcher et al. [37] & QA & No & 6 & Parallel & Phantom & $<0.04$ (rep. pos. acc \\
\hline Alnaghy et al. [38] & QA & No & 6 & Serial & Phantom & $<0.3$ (rep. pos. acc.) \\
\hline Shiinoki et al. [39] & QA & No & $6+3$ & Serial & Phantom & 0.11 (rep. pos. acc.) \\
\hline
\end{tabular}

Acc. refers to the end-to-end beam delivery accuracy for RT devices, patient positioning/motion tracking accuracy for patient couches/motion compensation, tracking accuracy for US motion tracking, and needle placement accuracy in the specified medium for BT.

compared to 4 DoF couches [47-49]. Therefore, most commercially available solutions, e.g., from Accuray, Elekta,
Varian, Leoni, or BEC can position the patient in $6 \mathrm{DoF}$. Another consideration is the higher maximum load for some 
of the robotic couches, e.g., the RoboCouch (Accuray, Sunnyvale, USA).

For systems that do not allow moving the beam, moving the couch also presents an alternative for motion compensation, compare Fig. 3. While good dosimetric accuracy has been reported [50,51], couch movement can cause unconscious physiological response. Still, Jöhl et al. found acceptable levels of stress in healthy participants [52]. The authors also studied requirements on the accuracy and dynamic properties for robotic couches to enable motion compensation and evaluated several commercially available models [53]. The idea to actively move the patient to align the target with the beam has also been studied in the context of cranial RS using conventional gantry based LINACs [19]. Using a parallel kinematics for $6 \mathrm{DoF}$ head motion, the authors intend to realize frameless and maskless RS.

For cranial interventions in particular, conventional nonrobotic methods involve fixing the patient's head either with a stereotactic frame or with an elastic mask. Belcher et al. addressed the $6 \mathrm{D}$ head motion compensation with a parallel kinematic design without using a mask or a frame [19]. They extended this work by proposing a custom soft robot with compliant components for more precise and comfortable motion compensation [20]. Furthermore, they introduced a control strategy for motion compensation to minimize total deviation from the target pose based on the dynamic properties of the specific robot used [21]. Here, they used the L-BFGS optimization algorithm to find trajectories that could keep head or prostate motion within $0.5-\mathrm{mm}$ deviation $100 \%$ of the time. Ostyn et al. studied the issue of interference of metallic components which can impact the dose delivery by attenuating the radiation. Therefore, they incorporated RT-compatible components close to the target while

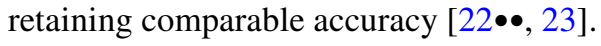

Recently, active couch motion has been considered to overcome limitations regarding the flexibility of dose delivery of gantry-based systems. The additional DoF for beam positioning relative to the patient can offer improved OAR sparing compared to a fixed couch position on gantry-based systems [54]. Examples for coordinated patient couch and gantry motion include dynamic or static positioning of the treatment couch, achieving non-coplanar treatment plans [55-59]. Commercial solutions are also available, e.g., the Elements Cranial SRS (Brainlab, Munich, Germany) or Hyperarc (Varian, Palo Alto, USA). However, new challenges arise with the coordinated motion since collision avoidance has to be ensured. Miao et al. presented a method for detecting collisions between gantry, couch, and patient [60].

Uncertainty in patient position can impact plan quality [61] and corresponds to higher margins when defining the PTV. Furthermore, any deviations not covered by the margins can lead to missing the targeted tissue during treatment.
To address this issue, Tsai et al. presented a system for validating treatment couch position without complicating the treatment workflow [62].

\section{Robotic Image Guidance}

Patient positioning and motion compensation rely largely on image data. Initially, computed tomography (CT) and sometimes also magnetic resonance imaging (MRI) are used to identify and delineate the PTV and the PRVs. The CT is also used to estimate the dose and to establish the optimization problem underlying inverse treatment planning. Hence, it is important to ensure the same alignment of beams and patient throughout the treatment to guarantee that the planned dose is delivered.

Most recent treatment systems use image guidance to match the actual patient pose with that in the planning CT. While MRI for pre- and intra-treatment has been integrated with LINACs, these systems remain relatively rare and more expensive. Moreover, the flexibility in positioning beams is further complicated by the MRI device. In contrast, X-ray image guidance is more widely used and often integrated with conventional gantry-based LINACs. Stereo X-ray imaging is also used with the CyberKnife and the VERO system to establish the target position. Furthermore, robotic C-arms have also been used for two-dimensional and cone beam $\mathrm{CT}$ imaging in RT. For example, a robotic system for CT imaging has been presented by Hua et al. [24]. Here, the authors mounted a $\mathrm{C}$-arm for cone beam imaging on a ceiling mounted robotic arm. This setup allowed imaging in the iso-center for fixed-beam proton therapy while using a 6 DoF patient couch for alignment.

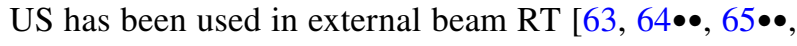
66]. Generally, two main applications can be distinguished: US guidance for inter-fractional patient setup and for intrafractional image guidance. The former ensures the correct position of the patient between fractions while the latter tracks the movement of the target during treatment to either pause treatment in case of large deviations or continually adapt the treatment plan according to target motion.

Inter-fractional US tracking has been commercially established by non-robotic systems like Clarity (Elekta, Stockholm, Sweden) [67], SonArray (Varian, Palo Alto, USA), and the B-Mode Acquisition and Targeting (BAT) system (Best Nomos, Pittsburgh, USA). The Clarity system can also be used for real-time intra-fractional target tracking, particularly during prostate cancer treatments. Generally, it is difficult to place the US probe such that the image quality is acceptable throughout the treatment and the beam delivery is not compromised. Hence, there is active research on the dosimetric impact of probe position if the probe is present during treatment as well as active robotic US for 
intra-fraction tracking. Since US probe and robot are generally not radio-translucent, care must be taken that treatment beams are not blocked. This can either be achieved by designing radiolucent components or by considering the ultrasound robot position during treatment planning.

Schlosser et al. presented a custom force-controlled robot for intra-fraction motion tracking with a $2 \mathrm{D}$ probe for gantry-based RT [25]. They also created a US visibility map based on the patient's CT scan to automatically find feasible US view ports. Furthermore, they determined if the robot and probe geometry interfered with medical devices, patient, and treatment beams and reported that in $60 \%$ of treatment plans, robot positions with no interference could be found.

Other groups used off-the-shelf robots for intra-fraction US tracking. Seitz et al. integrated robotic US into their custom framework [28]. They mounted a 2D curved array probe to an LBR iiwa (KUKA, Augsburg, Germany) and manually pre-positioned the probe either by hand-guiding or by selection of an imaging pose in the CT scan of the patient. They propose to perform a sweeping motion around the initial position while keeping a contact force of app. $5 \mathrm{~N}$.

Extending earlier work on 4D tracking [68], Ipsen et al. described a robotic system to automatically position the US transducer for intra-fraction motion tracking [26]. The system based on an LBR iiwa robot (KUKA, Augsburg, Germany) is designed for longer-term imaging and automatically maintains a contact force of app. $10 \mathrm{~N}$. Real-time volumetric ultrasound tracking was demonstrated for prostate and liver.

Sen and Bell et al. proposed cooperatively controlled robotic US for patient setup in RT [27, 69-71]. They first used a custom-built robot with 5 DoF but switched to a UR3 robot (Universal Robots, Odense, Denmark) because of simpler handling by the physician during setup. The authors increased repeatability of US probe placement by guiding the operator with haptic feedback to suitable imaging positions using optical markers.

To avoid beam blocking by the robot or US probe, either the robot position can be adjusted to not interfere with the treatment plan as Schlosser et al. have shown for gantrybased RT [25], or treatment planning can be adapted such that it considers the position of the radiopaque objects and blocked beams are removed. Especially for robotic RT and RS, the latter is necessary since the range of possible beam directions does not reliably permit options to place the robotic US without treatment plan interference. In previous works, we have developed methods for adjusting the treatment planning to that end and studied the effect of removed beams on the treatment plan quality [72•,73]. Furthermore, we have shown how to optimize robot positioning and treatment plan delivery when considering robotic US in combination with robotic beam delivery by the CyberKnife [74, 75].
Of particular interest for BT and proton therapy are mobile CT scanners due to their increased flexibility in positioning and a smaller footprint in the treatment room compared to conventional CT scanners. While the indication of mobile CT scanners is primarily in image-guided spine surgery, Chernavsky et al. assessed the Airo scanner (Brainlab, Munich, Germany) for image-guided BT [76]. Furthermore, Oliver et al. compared its performance to existing non-robotic CT scanners [77]. Both groups concluded that image quality was sufficient for their respective use case.

\section{Robotic Brachytherapy}

Robotic needle placement has been studied for more than 20 years and a variety of systems have been proposed, including for BT. Podder et al. give a comprehensive overview of systems and requirements for implementing robotic BT [78••]. A commercial system, First (Elekta, Stockholm, Sweden) [29], was introduced in 2002. It combines transrectal ultrasound (TRUS) with automatic needle placement. However, concerns were raised about the clinical outcome compared to conventional manual BT [79]. One general challenge in robotic BT is the safety of the system. Since the implantation of radioactive seeds is an invasive procedure, particular care has to be taken in order to verify the system's safe operation, compare Dai et al. for an overview of recent methods [80].

Several custom robotic systems have been proposed for BT needle placement. However, most systems position a needle guide or template and leave needle insertion to the physician. Dou et al. presented a robotic system for lung BT which aims to simplify the established clinical workflow by automatically placing the template at the position identified in the planning CT [31]. Their custom robot is fixed to the CT table and registered to the planning CT. Ma et al. [36] proposed a robotic system for template positioning with $\mathrm{CT}$ guidance. Their workflow involved an initial CT scan of patient and template to define the target position of the template. A second CT scan should verify the accuracy of the first needle before continuing to place the remaining needles. The authors reported a significant reduction in treatment time and improved needle placement accuracy compared to the conventional approach. The custom semi-automatic robotic system presented by Jiang et al. works similar to the First system [34]. It integrates TRUS and the template placement with a 3 DoF positioning platform, 2 DoF for US probe adjustment, and $4 \mathrm{DoF}$ for manual adjustment of the template.

Robotic systems also allow placing individual needles with different orientations without using a template. This flexibility has the potential of improving dose distributions. A system allowing individual needle placement is the 
Remebot system, which was evaluated by Fanhao et al. [33]. It is composed of an off-the-shelf 6 DoF robot on a moving platform and was originally designed as a stereotactic robot system for neurosurgery but has been shown to assist in head and neck BT by positioning the needle guide. For registration, the system uses optical markers and registers them to a pre-treatment MRI scan. As with similar systems, needle insertion is performed by the physician.

Some groups also studied automated needle insertion, e.g., to improve accuracy or to enable new applications of BT. For example, Lin et al. developed a custom robot for $\mathrm{BT}$ in the liver with $5 \mathrm{DoF}$ for positioning the needle guide and an actuator for needle insertion with an integrated force sensor [35]. They used artificial potential field-based motion planning for collision avoidance and an integrated US probe, mounted to the robot, for image guidance.

A novel approach for BT is the insertion of shielded needles to improve dose sparing of OARs. However, placement and rotation of multiple needles present a challenge. Adams et al. presented a mechanically robust robotic system which could deliver rotating shield BT treatments [30•]. Their custom 5 DoF robot with an additional angular needle drive could automatically deliver planned dose distributions for simulated treatments with acceptable accuracy.

Another less conventional application was reported by Wolfe et al. [81]. During laparoscopic robot-assisted resection of a urethral carcinoma with the da Vinci system (Intuitive Surgical, Sunnyvale, USA), the authors used the robotic system to deliver high-dose-rate $\mathrm{BT}$ to the resected area, as the initially planned SBRT was deemed infeasible due to close proximity to an OAR. At 9-month followup, no evidence of disease recurrence or metastases was found.

\section{Robotic Quality Assurance}

A further application of robotics in RT is to support quality assurance (QA), i.e., the routine tests and checks to verify that dose delivery is accurate. This can be particularly challenging for motion compensated treatments, where it is important, considering the impact of tracking errors on the dose distribution [61]. In one of the earlier works on QA for motion tracking in RT, Belcher et al. proposed to use a 6 DoF Stewart platform for motion trace reproduction in cranial RT [37]. The custom-designed robot achieved an RMSE of $0.04 \mathrm{~mm}$.

Alnaghy et al. proposed using a UR3 (Universal Robots, Odense, Denmark) to reproduce tumor motion traces [38]. They employed a polymethylmethacrylate phantom with embedded markers to implement a QA procedure for the tracking accuracy of the RT system. The authors reported a reproducibility of the motion traces of $0.3 \mathrm{~mm}$ and $0.5 \mathrm{deg}$.
Shiinoki et al. developed a patient-specific procedure for respiratory motion QA using a MZ07-1 robot (Nachi-Fujikoshi, Tokyo, Japan) [39]. Here, respiratory motion of the patient was recorded and a 3D printed model of the patient anatomy was placed inside a water basin. The basin was mounted to a robot and respiratory motion was simulated. The authors reported sufficient accuracy of the reproduced movement for QA purposes.

\section{Conclusion}

Robotic systems support or enable RT and RS in a variety of ways. From beam guidance and motion tracking to quality assurance, imaging, and BT, robotic systems can improve accuracy, safety, and flexibility compared to conventional systems. Recently, there has been encouraging progress in academia as well as in the industry towards further integration of robotic systems into the clinics.

While this review paper focused on the design and specifications of the robotic systems, multiple further interesting topics including dosimetry, treatment planning, and image processing are related to the development of robotic systems in RT and RS.

Funding Open Access funding enabled and organized by Projekt DEAL. The authors report grants from DFG (German Research Foundation) during execution of the study.

\section{Declarations}

Conflict of Interest The authors declare no competing interests.

Human and Animal Rights and Informed Consent This article does not contain any studies with human or animal subjects performed by any of the authors.

Open Access This article is licensed under a Creative Commons Attribution 4.0 International License, which permits use, sharing, adaptation, distribution and reproduction in any medium or format, as long as you give appropriate credit to the original author(s) and the source, provide a link to the Creative Commons licence, and indicate if changes were made. The images or other third party material in this article are included in the article's Creative Commons licence, unless indicated otherwise in a credit line to the material. If material is not included in the article's Creative Commons licence and your intended use is not permitted by statutory regulation or exceeds the permitted use, you will need to obtain permission directly from the copyright holder. To view a copy of this licence, visit http://creativecommons.org/licenses/by/4.0/.

\section{References}

Papers of particular interest, published recently, have been highlighted as:

- Of importance 
$\bullet$ Of major importance

1. The Global Cancer Observatory. All cancers fact sheet. Available from: https://gco.iarc.fr/today/data/factsheets/cancers/39All-cancers-fact-sheet.pdf (accessed 2021 Oct 21).

2. Baumann M, Krause M, Overgaard J, Debus J, Bentzen SM, Daartz J, et al. Radiation oncology in the era of precision medicine. Nat Rev Cancer. 2016;16(4):234-49. https://doi.org/10. 1038/nrc.2016.18.

3.•• Kilby W, Naylor M, Dooley JR, Maurer CR, Sayeh S. 2 - A Technical Overview of the CyberKnife System. In: Abedin-Nasab $\mathrm{MH}$, editor. Handbook of robotic and image-guided surgery. Amsterdam, Netherlands: Elsevier; 2020. 15-38. (A recent summary of the CyberKnife system. It contains a detailed description of all components which make up the robotic RT and RS system.)

4. Jaccard M, Durán MT, Petersson K, Germond J-F, Liger P, Vozenin M-C, et al. High dose-per-pulse electron beam dosimetry: commissioning of the Oriatron eRT6 prototype linear accelerator for preclinical use. Med Phys. 2018;45(2):863-74. https:// doi.org/10.1002/mp.12713.

5. Bourhis J, Sozzi WJ, Jorge PG, Gaide O, Bailat C, Duclos F, et al. Treatment of a first patient with FLASH-radiotherapy. Radiother Oncol. 2019;139:18-22. https://doi.org/10.1016/j. radonc.2019.06.019.

6. Miyabe Y, Sawada A, Takayama K, Kaneko S, Mizowaki T, Kokubo M, et al. Positioning accuracy of a new image-guided radiotherapy system. Med Phys. 2011;38(5):2535-41. https:// doi.org/10.1118/1.3578607.

7. Adler JR, Schweikard A, Achkire Y, Blanck O, Bodduluri M, $\mathrm{Ma} \mathrm{L}$, et al. Treatment planning for self-shielded radiosurgery Cureus. 2017;9(9):e1663. https://doi.org/10.7759/cureus.1663.

8. Weidlich GA, Schneider MB, Simcic V, Oostman Z, Adler JR. Self-shielding for the ZAP-X®: revised characterization and evaluation. Cureus. 2021;13(3):e13660. https://doi.org/10.7759/ cureus.13660.

9. Weidlich GA, Bodduluri M, Achkire Y, Lee C, Adler JR. Characterization of a novel 3 megavolt linear accelerator for dedicated intracranial stereotactic radiosurgery. Cureus. 2019;11(3):e4275. https://doi.org/10.7759/cureus.4275.

10. Soiort - S.I.T. Sordina IORT Technologies S.p.A. Available from: https://www.soiort.com/ (accessed 2021 Nov 1).

11. Noh SY, Jeong K, Seo Y, Kim C, Park J, Choi YR, et al. Development of a prototype robotic system for radiosurgery with upper hemispherical workspace. J Healthc Eng. 2017;2017:4264356. https://doi.org/10.1155/2017/4264356.

12. Accuray. cyberknife-treatment-delivery-system_-technical-specifications. Available from: https://www.accuray.com/wp-content/ uploads/cyberknife-treatment-delivery-system_-technical-speci fications.pdf (accessed 2021 Oct 20).

13. Riis HL, Zimmermann SJ. Elekta Precise Table characteristics of IGRT remote table positioning. Acta Oncol. 2009;48(2):267-70. https://doi.org/10.1080/02841860802311007.

14. Elekta Instrument AB Stockholm. Radiation Oncology Accessories Catalog. Available from: https://ecatalog.elekta.com/oncol ogy/archive/files/22384/2013-radiation-oncology-accessoriescatalog-pdf.aspx (accessed 2021 Oct 20).

15. Tiegel G. Couch6doF. Available from: http://photonix.com. ar/wp-content/uploads/2018/10/perfect-pitch-couch-6d.pdf (accessed 2021 Oct 20).

16. Leoni. leoni_orion_precision_patient_positioning_system. Available from: https://publications.leoni.com/fileadmin/ healthcare/publications/data_sheets/leoni_orion_precision patient_positioning_system.pdf?1540201303 (accessed 2021 Oct 20).
17. BEC GmbH - robotic solutions. Medical Robotics - BEC $\mathrm{GmbH}$ - robotic solutions. Available from: https://www.b-e-c. de/us/medical (accessed 2021 Oct 20).

18. Civco. ProturaFAQ. Available from: https://civcort.com/ro/resou rces/faq-stat-sheets/ProturaFAQ.pdf (accessed 2021 Nov 1).

19. Belcher AH, Liu X, Chmura S, Yenice K, Wiersma RD. Towards frameless maskless SRS through real-time 6 DoF robotic motion compensation. Phys Med Biol. 2017;62(23):9054-66. https:// doi.org/10.1088/1361-6560/aa93d2.

20. Ogunmolu O, Liu X, Gans N, Wiersma RD. Mechanism and model of a soft robot for head stabilization in cancer radiation therapy. IEEE Int Conf Robot Autom. 2020;2020:4609-15. https://doi.org/10.1109/icra40945.2020.9197007.

21. Liu X, Wiersma RD. Optimization based trajectory planning for real-time $6 \mathrm{DoF}$ robotic patient motion compensation systems. PLoS ONE. 2019;14(1):e0210385. https://doi.org/10. 1371/journal.pone.0210385.

$22 . \bullet$ Ostyn M, Wang S, Kim Y-S, Kim S, Yeo W-H. Radiotherapycompatible robotic system for multi-landmark positioning in head and neck cancer treatments. Sci Rep. 2019;9(1):14358. https://doi.org/10.1038/s41598-019-50797-7. (This paper describes a system which offers 6 DoF patient positioning while using radio-compatible materials. Therefore, it can more readily be included in clinical practice.)

23. Ostyn M, Dwyer T, Miller M, King P, Sacks R, Cruikshank R, et al. An electromechanical, patient positioning system for head and neck radiotherapy. Phys Med Biol. 2017;62(18):7520-31. https://doi.org/10.1088/1361-6560/ aa86e3.

24. Hua C, Yao W, Kidani T, Tomida K, Ozawa S, Nishimura $\mathrm{T}$, et al. A robotic $\mathrm{C}$-arm cone beam $\mathrm{CT}$ system for imageguided proton therapy: design and performance. Br J Radiol. 2017;90(1079):20170266. https://doi.org/10.1259/bjr.20170266.

25. Schlosser J, Gong RH, Bruder R, Schweikard A, Jang S, Henrie $\mathrm{J}$, et al. Robotic intrafractional US guidance for liver SABR: System design, beam avoidance, and clinical imaging. Med Phys. 2016;43(11):5951. https://doi.org/10.1118/1.4964454.

26. Ipsen S, Wulff D, Kuhlemann I, Schweikard A, Ernst F. Towards automated ultrasound imaging-robotic image acquisition in liver and prostate for long-term motion monitoring. Phys Med Biol. 2021;66(9):94002. https://doi.org/10.1088/1361-6560/abf277.

27. Şen HT, Cheng A, Ding K, Boctor E, Wong J, Iordachita I, et al. Cooperative control with ultrasound guidance for radiation therapy. Front Robot AI. 2016;3:49. https://doi.org/10.3389/frobt. 2016.00049.

28. Seitz PK, Baumann B, Johnen W, Lissek C, Seidel J, Bendl R. Development of a robot-assisted ultrasoundguided radiation therapy (USgRT). Int J Comput Assist Radiol Surg. 2020;15(3):491-501. https://doi.org/10.1007/ s11548-019-02104-y.

29. Rivard MJ, Evans D-AR, Kay I. A technical evaluation of the Nucletron FIRST system: conformance of a remote afterloading brachytherapy seed implantation system to manufacturer specifications and AAPM Task Group report recommendations. J Appl Clin Med Phys. 2005 6(1):22-50. https://doi.org/10.1120/jacmp. v6i1.1985.

30.• Adams Q, Hopfensperger KM, Kim Y, Wu X, Flynn RT. 169 Yb-based rotating shield brachytherapy for prostate cancer. Med Phys. 2020;47(12):6430-9. https://doi.org/10.1002/mp.14533. (This paper introduces a system to deliver a novel type of rotating shield BT allowing for improved dose distribution. It represents an interesting contribution to the field of robotic BT.)

31. Dou H, Jiang S, Yang Z, Sun L, Ma X, Huo B. Design and validation of a CT-guided robotic system for lung cancer 
brachytherapy. Med Phys. 2017;44(9):4828-37. https://doi.org/ 10.1002/mp.12435.

32. Ma X, Yang Z, Jiang S, Huo B, Cao Q, Chai S, et al. Effectiveness and safety of a robot-assisted $3 \mathrm{D}$ personalized template in 125I seed brachytherapy of thoracoabdominal tumors. J Contemp Brachytherapy. 2018;10(4):368-79. https://doi.org/10. 5114/jcb.2018.77957.

33. Fanhao M, Xiaodong X, Bo Q, Lejun X, Yu S, Liang Z, et al. A new multimodal, image-guided, robot-assisted, interstitial brachytherapy for the treatment of head and neck tumorsa preliminary study. Int $\mathbf{J}$ Med Robot Comput Assist Surg. 2020;16(5):1-5. https://doi.org/10.1002/rcs.2133.

34. Jiang S, Yang Y, Yang Z, Zhang Z, Liu S. Design and experiments of ultrasound image-guided multi-DOF robot system for brachytherapy. Trans Tianjin Univ. 2017;23(5):479-87. https:// doi.org/10.1007/s12209-017-0067-9.

35. Lin X, Zhou S, Wen T, Jiang S, Wang C, Chen J. A novel multi-DoF surgical robotic system for brachytherapy on liver tumor: design and control. Int J Comput Assist Radiol Surg. 2021;16(6):1003-14. https://doi.org/10.1007/ s11548-021-02380-7.

36. Ma X, Yang Z, Jiang S, Zhang G, Chai S. A novel auto-positioning method in Iodine-125 seed brachytherapy driven by preoperative planning. J Appl Clin Med Phys. 2019;20(6):2330. https://doi.org/10.1002/acm2.12591.

37. Belcher AH, Liu X, Grelewicz Z, Pearson E, Wiersma RD. Development of a $6 \mathrm{DOF}$ robotic motion phantom for radiation therapy. Med Phys. 2014;41(12):121704. https://doi.org/10. 1118/1.4900828.

38. Alnaghy S, Kyme A, Caillet V, Nguyen DT, O'Brien R, Booth $\mathrm{JT}$, et al. A six-degree-of-freedom robotic motion system for quality assurance of real-time image-guided radiotherapy. Phys Med Biol. 2019;64(10):105021. https://doi.org/10.1088/ 1361-6560/ab1935.

39. Shiinoki T, Fujii F, Fujimoto K, Yuasa Y, Sera T. A novel dynamic robotic moving phantom system for patient-specific quality assurance in real-time tumor-tracking radiotherapy. J Appl Clin Med Phys. 2020;21(7):16-28. https://doi.org/10. 1002/acm2.12876.

40. Guthrie BL, Adler JR. Computer-assisted preoperative planning, interactive surgery, and frameless stereotaxy. Clin Neurosurg. 1992;38:112-31.

41. Schlaefer A, Schweikard A. Stepwise multi-criteria optimization for robotic radiosurgery. Med Phys. 2008;35(5):2094103. https://doi.org/10.1118/1.2900716. (This paper is a detailed description of the optimization problem which is the inverse planning problem for robotic RS. It describes the different competing objectives considered in treatment planning.)

42. Kearney V, Cheung JP, McGuinness C, Solberg TD. CyberArc: a non-coplanar-arc optimization algorithm for CyberKnife. Phys Med Biol. 2017;62(14):5777-89. https://doi.org/10.1088/13616560/aa6f92.

43. Kearney V, Descovich M, Sudhyadhom A, Cheung JP, McGuinness C, Solberg TD. A continuous arc delivery optimization algorithm for CyberKnife m6. Med Phys. 2018. https://doi.org/ 10.1002/mp.13022.

44. Schweikard A, Shiomi H, Adler J. Respiration tracking in radiosurgery. Med Phys. 2004;31(10):2738-41. https://doi.org/10. 1118/1.1774132.

45. Ernst F, Dürichen R, Schlaefer A, Schweikard A. Evaluating and comparing algorithms for respiratory motion prediction. Phys Med Biol. 2013;58(11):3911-29. https://doi.org/10.1088/00319155/58/11/3911.

46.• Hiraoka M, Mizowaki T, Matsuo Y, Nakamura M, Verellen D. The gimbaled-head radiotherapy system: rise and downfall of a dedicated system for dynamic tumor tracking with realtime monitoring and dynamic WaveArc. Radiother Oncol. 2020;153:311-8. https://doi.org/10.1016/j.radonc.2020.07.002. (A description of the reasons for the recent discontinuing of the Vero system. As one of the few commercial robotic RT systems, the insights are interesting also for the future of robotic $R T$ in general.)

47. Liu RJ, Yang SX, Neylon J, Hall MD, Dandapani S, Vora N, et al. Residual setup errors in cranial stereotactic radiosurgery without six degree of freedom robotic couch: frameless versus rigid immobilization systems. J Appl Clin Med Phys. 2020;21(3):87-93. https://doi.org/10.1002/acm2.12828.

48. Rodrigues MF, Veen S, van Egmond J, van Hameren M, van Oorschot T, de Vet S, et al. The influence of a six degrees of freedom couch and an individual head support in patient positioning in radiotherapy of head and neck cancer. Phys Imaging Radiat Oncol. 2019;11:30-3. https://doi.org/10.1016/j.phro.2019.07. 001.

49. Sagawa T, Ohira S, Ueda Y, Akino Y, Mizuno H, Matsumoto $\mathrm{M}$, et al. Dosimetric effect of rotational setup errors in stereotactic radiosurgery with HyperArc for single and multiple brain metastases. J Appl Clin Med Phys. 2019;20(10):84-91. https:// doi.org/10.1002/acm2.12716.

50. Zhang L, LoSasso T, Zhang P, Hunt M, Mageras G, Tang G. Couch and multileaf collimator tracking: a clinical feasibility study for pancreas and liver treatment. Med Phys. 2020;47(10):4743-57. https://doi.org/10.1002/mp.14438.

51. Ehrbar S, Schmid S, Jöhl A, Klöck S, Guckenberger M, Riesterer $\mathrm{O}$, et al. Comparison of multi-leaf collimator tracking and treatment-couch tracking during stereotactic body radiation therapy of prostate cancer. Radiother Oncol. 2017;125(3):445-52. https://doi.org/10.1016/j.radonc.2017.08.035.

52. Jöhl A, Bogowicz M, Ehrbar S, Guckenberger M, Klöck S, Meboldt M, et al. Unconscious physiological response of healthy volunteers to dynamic respiration-synchronized couch motion. Radiat Oncol. 2017;12(1):189. https://doi.org/10.1186/ s13014-017-0925-6.

53. Jöhl A, Ehrbar S, Guckenberger M, Klöck S, Mack A, Meboldt $\mathrm{M}$, et al. The ideal couch tracking system-requirements and evaluation of current systems. J Appl Clin Med Phys. 2019;20(10):152-9. https://doi.org/10.1002/acm2.12731.

54. Amaya D, Shinde A, Wohlers C, Wong KCC, Novak J, Neylon $\mathrm{J}$, et al. Dosimetric comparison of multiple vs single isocenter technique for linear accelerator-based stereotactic radiosurgery: the importance of the six degree couch. J Appl Clin Med Phys. 2021;22(6):45-9. https://doi.org/10.1002/acm2.13286.

55. Smyth G, Evans PM, Bamber JC, Bedford JL. Recent developments in non-coplanar radiotherapy. Br J Radiol. 2019;92(1097):20180908. https://doi.org/10.1259/bjr.20180908.

56. MacDonald RL, Syme A, Little B, Ward L, Thomas CG. Toward the combined optimization of dynamic axes (CODA) for stereotactic radiotherapy and radiosurgery using fixed couch trajectories. Med Phys. 2020;47(2):307-16. https://doi.org/10.1002/mp. 13887.

57. Subramanian VS, Subramani V, Chilukuri S, Kathirvel M, Arun G, Swamy ST, et al. Multi-isocentric $4 \pi$ volumetric-modulated arc therapy approach for head and neck cancer. J Appl Clin Med Phys. 2017;18(5):293-300. https://doi.org/10.1002/acm2.12164.

58. Wilson B, Otto K, Gete E. A simple and robust trajectory-based stereotactic radiosurgery treatment. Med Phys. 2017;44(1):240 8. https://doi.org/10.1002/mp.12036.

59. Lyu Q, Neph R, Yu VY, Ruan D, Boucher S, Sheng K. Manyisocenter optimization for robotic radiotherapy. Phys Medi Biol. 2020;65(4):45003. https://doi.org/10.1088/1361-6560/ab63b8.

60. Miao J, Niu C, Liu Z, Tian Y, Dai J. A practical method for predicting patient-specific collision in radiotherapy. J Appl Clin 
Med Phys. 2020;21(8):65-72. https://doi.org/10.1002/acm2. 12915.

61. Tanaka Y, Oita M, Inomata S, Fuse T, Akino Y, Shimomura $\mathrm{K}$. Impact of patient positioning uncertainty in noncoplanar intracranial stereotactic radiotherapy. J Appl Clin Med Phys. 2020;21(2):89-97. https://doi.org/10.1002/acm2.12820.

62. Tsai P, Liu C, Kahler DL, Li JG, Lu B, Yan G. A self-checking treatment couch coordinate calculation system in radiotherapy. $\mathrm{J}$ Appl Clin Med Phys. 2020;21(1):43-52. https://doi.org/10.1002/ acm2.12771.

63. Camps SM, Fontanarosa D, With PH de, Verhaegen F, Vanneste BG. The use of ultrasound imaging in the external beam radiotherapy workflow of prostate cancer patients. BioMed Res Int. 2018;2018.

64.•• Davide Fontanarosa, Skadi van der Meer, Jeffrey Bamber, Emma Harris, Tuathan O'Shea, Frank Verhaegen. Review of ultrasound image guidance in external beam radiotherapy: I. Treatment planning and inter-fraction motion management. Phys Med Biol. 2015;60(3) R77. https://doi.org/10.1088/0031-9155/60/3/ R77.This review gives an extensive overview over the use of US in external RT with respect to treatment planning and inter-fraction motion.

65.•• O'Shea T, Bamber J, Fontanarosa D, van der Meer S, Verhaegen F, Harris E. Review of ultrasound image guidance in external beam radiotherapy part II: intra-fraction motion management and novel applications. Phys Med Biol. 2016;61(8):R90. https:// doi.org/10.1088/0031-9155/61/8/R90. (This review continues the overview of US in external RT with a summary of US in intra-fraction guidance.)

66. Bertholet J, Knopf A, Eiben B, McClelland J, Grimwood A, Harris $\mathrm{E}$, et al. Real-time intrafraction motion monitoring in external beam radiotherapy. Phys Med Biol. 2019;64(15):15TR01. https://doi.org/10.1088/1361-6560/ab2ba8.

67. Grimwood A, McNair HA, O'Shea TP, Gilroy S, Thomas K, Bamber JC, et al. In vivo validation of Elekta's Clarity Autoscan for ultrasound-based intrafraction motion estimation of the prostate during radiation therapy. Int J Radiat Onc Biol Phys. 2018;102(4):912-21. https://doi.org/10.1016/j.ijrobp.2018.04. 008.

68. Ipsen S, Bruder R, O’Brien R, Keall PJ, Schweikard A, Poulsen PR. Online 4D ultrasound guidance for real-time motion compensation by MLC tracking. Med Phys. 2016;43(10):5695. https://doi.org/10.1118/1.4962932.

69. H. T. Sen, M. A. L. Bell, I. Iordachita, J. Wong, P. Kazanzides. A cooperatively controlled robot for ultrasound monitoring of radiation therapy. In: IEEE Int Conf Intell Robots Syst; 2013. 3071-6.

70. Lediju Bell MA, Sen HT, Iordachita I, Kazanzides P, Wong J. In vivo reproducibility of robotic probe placement for a novel ultrasound-guided radiation therapy system. J Med Imaging. 2014;1(2):25001. https://doi.org/10.1117/1.JMI.1.2.025001.

71. Sen HT, Bell MAL, Zhang Y, Ding K, Boctor E, Wong J, et al. System integration and in vivo testing of a robot for ultrasound guidance and monitoring during radiotherapy. IEEE Trans Biomed Eng. 2017;64(7):1608-18. https://doi.org/10.1109/ TBME.2016.2612229.

72. Gerlach S, Kuhlemann I, Jauer P, Bruder R, Ernst F, Fürweger C, et al. Robotic ultrasound-guided SBRT of the prostate: feasibility with respect to plan quality. Int J Comput Assist Radiol Surg. 2017;12(1):149-59. https://doi.org/10.1007/s11548-016-1455-7. (This paper presents an approach to integrate robotic US in robotic RT by adapting treatment planning. It shows feasibility with respect to plan quality.)

73. Gerlach S, Kuhlemann I, Ernst F, Fürweger C, Schlaefer A. Impact of robotic ultrasound image guidance on plan quality in SBRT of the prostate. Brit J Radiol. 2017;90(1078):20160926. https://doi.org/10.1259/bjr.20160926.

74. Schlüter M, Gerlach S, Fürweger C, Schlaefer A. Analysis and optimization of the robot setup for robotic-ultrasound-guided radiation therapy. Int $\mathrm{J}$ Comput Assist Radiol Surg. 2019;14(8):1379-87. https://doi.org/10.1007/ s11548-019-02009-w.

75. Schlüter M, Fürweger C, Schlaefer A. Optimizing robot motion for robotic ultrasound-guided radiation therapy. Phys Med Biol. 2019;64(19). https://doi.org/10.1088/1361-6560/ab3bfb.

76. Chernavsky NE, Morcos M, Wu P, Viswanathan AN, Siewerdsen $\mathrm{JH}$. Technical assessment of a mobile CT scanner for imageguided brachytherapy. J Appl Clin Med Phys. 2019;20(10):187200. https://doi.org/10.1002/acm2.12738.

77. Oliver JA, Zeidan OA, Meeks SL, Shah AP, Pukala J, Kelly P, et al. The Mobius AIRO mobile CT for image-guided proton therapy: characterization \& commissioning. J Appl Clin Med Phys. 2017;18(3):130-6. https://doi.org/10.1002/acm2.12084.

78. • Podder TK, Beaulieu L, Caldwell B, Cormack RA, Crass JB, Dicker AP, et al. AAPM and GEC-ESTRO guidelines for imageguided robotic brachytherapy: report of Task Group 192. Med Phys. 2014;41(10):101501. https://doi.org/10.1118/1.4895013. (This review contains a description of published BT systems as well as guidelines and requirements for future systems.)

79. Genebes C, Filleron T, Graff P, Jonca F, Huyghe E, Thoulouzan $\mathrm{M}$, et al. Conventional versus automated implantation of loose seeds in prostate brachytherapy: analysis of dosimetric and clinical results. Int J Radiat Oncol Biol Phys. 2013;87(4):651-8. https://doi.org/10.1016/j.ijrobp.2013.08.010.

80. Dai X, Zhang Y, Jiang J, Li B. Image-guided robots for low dose rate prostate brachytherapy: perspectives on safety in design and use. Int J Med Robot Comput Assist Surg. 2021;17(3):e2239. https://doi.org/10.1002/rcs.2239.

81. Wolfe AR, Grecula JC, Blakaj D, Wald P, Carlson M, Woods $\mathrm{K}$, et al. Robotic-assisted intraoperative high-dose rate remote brachytherapy following laparoscopic robotic-assisted resection of pelvic recurrence of urethral carcinoma. Adv Radiat Oncol. 2019;4(3):443-6. https://doi.org/10.1016/j.adro.2019.03.006. 